\section{Hiatus leucaemicus}

H. Baum

Institut für Laboratoriumsmedizin, Mikrobiologie und Blutdepot, Regionale Kliniken Holding RKH GmbH, Ludwigsburg, Deutschland

Englischer Begriff hiatus leucaemicus

Definition Auftreten einer Blastenpopulation neben reifen Granulozyten im peripheren Blut. Zwischenstufen der Myelopoese sind dabei nicht nachweisbar.

Beschreibung Der Hiatus leucaemicus beschreibt eine typische Veränderung der Leukozytenzusammensetzung im peri- pheren Blut bei Patienten mit einer akuten Leukämie. Neben einer meist kleinen Population an reifen neutrophilen Granulozyten ist nur eine größere Population an unreifen, blastären Zellen nachweisbar. Nicht nachweisbar sind die Zwischenstufen der normalen Myelopoese, also $>$ Promyelozyt, \ Myelozyten, - Metamyelozyten und stabförmige Granulozyten ( $\triangleright$ Granulozyten, stabkernige).

\section{Literatur}

Begemann M (1993) Reaktive Veränderungen der weißen Blutkörperchen und des lymphoretikulären Systems. In: Begemann H, Rastetter J (Hrsg) Klinische Hämatologie, 4. Aufl. Georg Thieme Verlag, Stuttgart, S 441-442 